\title{
LEXICAL FUNCTIONS AND MACHINE TRANSLATION
}

\author{
Dirk Heylen, Kerry G. Maxwell and Marc Verhagen \\ OTS, Trans 10, 3512 JK Utrecht, Netherlands \\ CLMT Group, Essex University, Colchester, Essex CO4 3SQ, England \\ email: heylen@let.ruu.nl, kerry@essex.ac.uk, verhm@essex.ac.uk
}

This paper discusses the lexicographical concept of lexical functions (Mel'čuk and Žolkovsky, 1984) and their potential exploitation in the development of a machine translation lexicon designed to handle collocations. We show how lexical functions can be thought to reflect cross-linguistic meaning concepts for collocational structures and their translational equivalents, and therefore suggest themselves as some kind of languageindependent semantic primitives from which translation strategies can be developed. ${ }^{1}$

\section{Description of the Problem}

Collocations present specific problems in translation, both in human and automatic contexts. If we take the construction heavy smoker in English and attempt to translate it into French and German, we find that a literal translation of heavy yields the wrong result, since the concept expressed by the adjective (something like 'excessive') is translated by grand (large) in French and stark (strong) in German. We observe then that in some sense the adjectives stark, grand and heavy are equivalent in the collocational context, but that this is of course not typically the case in other contexts, of grande boite, starke Schachtel and heavy box, where the adjectives could hardly be viewed as equivalent. It seems then that adjectives which are not literal translations of one another may share meaning properties specifically in the collocational context.

How then can we specify this special equivalence in the machine translation dictionary? The answer seems to lie in addressing the concept which underlies the union of adjective and noun in these three cases, i.e., intensification, and hence establish a single meaning representation for the adjectives which can be viewed as an interlingual pivot for translation.

Collocations have been studied by computational linguists in different contexts. For instance, there is a substantial body of papers on the extraction of "frequently co-occurring words" from corpora using statistical methods (e.g., (Choueka et al., 1983), (Church and Hanks, 1989), (Smadja, 1993) to list only a few). These authors focus on techniques for providing material that can be used in other processing tasks such as

\footnotetext{
${ }^{1}$ The research reported in this paper was undertaken as the project "Collocations and the Lexicalisation of Semantic Operations" (ET10/75). Financial contributions were by the Commission of the European Community, Association Suissetra (Geneva) and Oxford University Press.
}

word sense disambiguation, information retrieval, natural language generation and so on. Also, the use of collocations in different applications has been discussed by various authors ((McRoy, 1992), (Pustejovsky et al., 1992), (Smadja and McKcown, 1990) etc.). However, collocations are not only considered useful, but also a problem both in certain applications (c.g. generation, (Nirenburg et al., 1988), machine translation, (Heid and Raab, 1989)) and from a more theoretical point of view (c.g. (Abeillé and Schabes, 1989), (Krenn and Erbach, to appear)).

We have been concerned with investigating the lexical functions (LFs) of Mel'čuk (Mel'čuk and Žolkovsky, 1984) as a candidate interlingual device for the translation of adjectival and verbal collocates. Our work is related to research by (Heid and Raab, 1989). In some respects it is an extension of some of their suggestions. Our work differs from theirs in scope and also in the exploration of various other directions.

\section{Representation}

The use we make of lexical functions as interlingual representations, does not respect their original Mel'čukian interpretation. Furthermore, we have transferred them from their context in the Mcaning-Text Theory to a different theoretical setting. We have embedded the concept in an HPSG-like grammar theory. ${ }^{2}$ In this section we review this operation. I'irst we consider the features of Mel'čuk's treatment that we have wanted to preserve. Next we show how they have been imported into the HPSG framework.

\subsection{Collocations and LFs}

In Mel'čuk's Explanatory Combinatory Dictionary (ECD, see (Mel'čuk et al, 1984)), expressions such as une ferme intention, une résistance acharnée, un argument de poids, un bruit infernal and donner une leçon, faire un pas, commetre un crime are described in the lexical combinatorics zone. These "expressions plus ou moins figées" will be called 'collocations'. They are considered to consist of two parts - the base and the collocate. In the examples above, the nouns are the bases and the adjectives and the verbs are the collocates. The idea that all adjective collocates and all the verb

\footnotetext{
${ }^{2}$ Head Driven Phrase Struclute grammar, see (Pollard and Sag, 1987), (Pollard and Sigg, to appear). For another treatment of collocations in HPSG, see (Kremn and Irbach, to appear).
} 
collocates share an important meaning component --. roughly paraphrasable as intense and do respectively -and the fact that the adjectives and verbs are not interchangeable but are restricted with this meaning to the accompanying nouns, is coded in the dictionary using lexical functions (in this case Magn and Oper).

Each article in the ECD describes what is called a 'lexeme': a word in some specific reading. In the lexical combinatorics zone, we find a list of the lexical functions that are relevant to this particular lexeme. Each lexical function is followed by one or more lexemes (the result or value of the function applied to the head word). The idea is that cach combination of the argument with one of the values of the function forms a collocation in our terminology. The argument corresponds to the base and each value is a collocate. The following features of this representation are important to us.

- $\Upsilon$ exical functions are used to represent an important syntactico-semantic relation between the base and the collocate.

- The restricted combinatorial potential of the collocate lexeme is accounted for by listing it at each base with which it can occur.

The second of these characteristics points out that the collocational restriction is scen as a purely lexical, idiosyncratic one: all collocations are explicitly listed.

One other aspect of collocations which we have to deal with is the relation between the collocate lexeme and its freely occurring counterpart. Collocate lexemes often differ in some respects from their literal variants while sharing other properties. Mel'cuk deals with this by including in the ECD an entry for the free variant and putting the collocate-specific information in the entry for the base (with the result of the lexical functions). The full entry of the collocate is the result of taking the entry for the free variant and overwriting it with the information provided at the base.

\subsection{Collocations in HPSG}

The three aspects of Mel'čuk's analysis we wanted to encode in HPSG were the following.

- Coding the base-collocate relation in the lexicon.

- Choosing the level at which lexical functions will be situated.

- Relating the collocate information to the free variant entry.

We have provided straightforward solutions to these problems. For the first problem we have taken over the LCD architecture rather directly, by creating a dedicated 'collocates' field in the entry for bases which contains all the relevant collocates. As far as the second problem is concerned, the obvious place to put lexical functions is in the semantic representation provided by HPSG. There are various reasons for this. One is that I I's are used in the deep syntax level in Mel'čuk's model, a level oriented towards meaning. Another reason is that this level seems most appropriate to be used in transfer/translation and because we want to use lexical functions in transfer, this is where they should be. In contrast to the ECD, the meaning of the collocate is represented by the lexical function only.

The following is an example of the entry for criticism with the cncoding of strong as a collocate. ${ }^{3}$ We use SEM_IND as an abbreviation for the feature path SLM.CONT.INI).

$$
\left[\begin{array}{ll}
\text { PHON } & \text { criticism } \\
\text { SEM_IND } & {\left[\begin{array}{l}
\text { VAR }[1] \\
\text { REST }\{\text { criticism[1]\} }\}
\end{array}\right]} \\
\text { COLLS } & \left\{\left[\begin{array}{l}
\text { \$strong } \\
\text { SEM_IND }\left[\begin{array}{ll}
\text { VAR } & 1 \\
\text { RLST } & \{\text { Magn }[1]\}\}
\end{array}\right]
\end{array}\right]\right\}
\end{array}\right]
$$

Just as in the ECD the base contains a specific zone in which the collocates are listed. In our case, the feature 'COLLs' has a set of lexical entries as its value.

Each collocate subentry bears the value of the lexical function in its semantics field. In this representation the lexical function is chosen as the real semantic value of the collocate. One should read the feature structure as specifying that the semantics of strong (as a collocate) is the predicate Magn ( 1$]$.

The collocate subentry only provides partial information. In fact, it provides only the information that is specitic to the occurrence of strong in its combination with criticism. In this case only the semantics is given. We further assume that the lexicon also contains a 'super-entry' which provides all the information that is shared by all the different occurrences of strong. This entry is where the variable \$strong points to. Of course, other architectures that try to avoid redundant specification of information are equally possible. For instance if one assumes a mechanism of default unification, one can have \$strong refer to the full entry describing 'strong' in say its ordinary use, and have the values that are particular to the collocational strong overwrite the values provided in the ordinary entry, as in Mel'čuk's proposal.

Collocations, Rules and Principles So far, we have not specified in what way one gets from the lexical cntries for the base and the collocate to the representation of the collocational expression.

In HPSG, the descriptions of complex expressions are constrained by principles. We will assume that collocations are subject to the same constraints. The ordinary rules of combination (combining adjectives and nouns, for instance) thus account for most of the properties of the collocational combination. However, we are still left with the typical collocational restriction' which needs to be accounted for.

We have therefore added a principle which says that constructions that are analysed as collocations (indicated by the type COLLOCATION) are cither head-adjunct structure or head-complement structures with specific restrictions holding between the head and the adjunct or

\footnotetext{
${ }^{3}$ Notice that here we use a simple version of HPSG based on (Pollard and Sag, 1987) whereas the actual implementation was based on (Pollard and Saig, to appear).
} 
the head and the complement respectively. Let's consider the former case ${ }^{4}$, illustrated by the heavy smoker example. The adjunct daughter will contain the adjective collocate. In such collocational constructions the collocate adjuncts have to be 'licensed' by the noun or the head daughter. This is implemented by requiring that the collocates field (COI.LS) of the head daughter contains a reference to a lexical entry that is compatible with the adjunct daughter. In the literal reading of an expression such as heavy smoker, the phrase will not be analysed as a COLLOCATION and the principle does not apply.

$$
\begin{aligned}
& {[]_{\text {COLLOCATION }} \Rightarrow} \\
& {\left[\begin{array}{l}
\text { HEAD_ITR } \\
\text { ADJ_ITRS COLLS }
\end{array}[\ldots[1] \ldots\}\right]} \\
& {\left[\begin{array}{ll}
\text { HEAD_DTR } & 1 \text { COLLOCATE } \\
\text { COMP.DTRS } & <\ldots[\text { COLLS }[\ldots[1] \ldots\}] \ldots>
\end{array}\right]}
\end{aligned}
$$

\section{Issues in Translation}

The project has tried to investigate the use of lexical functions as an interlingual device, i.e., one which is shared by the semantic representations of collocations in the language pairs ${ }^{5}$.

The typing of a collocation with such a function opens up the way to a treatment of collocations inside a given language module and hence to a substantial reduction in the number of collocations explicitly handled in the multilingual transfer dictionary. The existence of a collocation function is established during analysis. This information is used to generate the correct translation in the target language. To illustrate, the English analysis module might analyse (1) as (2). The transfer module maps (2) onto (3) which is then synthesised by the lirench module to (4)

(1) heavy smoker $\rightarrow$ (2) Magn(smoker) $\rightarrow$

(3) Magn(fumeur) $\rightarrow$ (4) grand fumeur

The example points out that the translation strategy is a mixture of transfer and interlingua. The bases are transferred but the representation of the collocate is shared between the source and the target representation. This treatment of collocations rests, among others, on the assumptions that there are only a limited number of lexical functions, that lexical functions can be assigned consistently, that all (or a significant number of) collocations realise a lexical function, that lexical functions are not restricted to particular languages, etc. In the following paragraph we present an outline of the translation process. Next, we discuss some of the problems which follow from our approach and we propose some ways to solve them.

\footnotetext{
${ }^{4}$ To illustrate the case of head-complement structures one could take some support verb construction (also called light verb cons(ruction).

${ }^{5}$ For another application of LFs in a multilingual NLP context sec (Heid and Ratab, 1989). For other treatments of collocations in language generation see (Nirenburg et al., 1988) and (Smadja and McKeown, 1990)
}

\subsection{Lexical Functions as Interlingua}

It was assumed that the starting point for transfer is the semantic representation of the phrase. Using a semantic representation as input to transfer implies that we relate semantic values of words and phrases. For our purposes this is very satisfying since we will now be using the semantics of collocates instead of their orthography, in other words: we use lexical functions and abstract away from the particular realisation of a collocate in a particular language.

We now statc the relation between the semantic representations of the source language and target language. The semantic relation between the phrase heavy smoker and its French counterpart can be made explicit in the following bilingual sign:

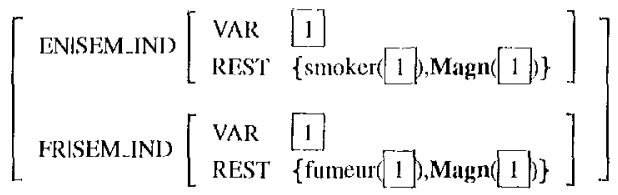

Typically, the lexicon will contain a bilingual sign for each possible value of RLIN. Thus, for translating heavy smoker into grand fumeur we will need the obvious entry for smoker-fumeur plus the entry below:

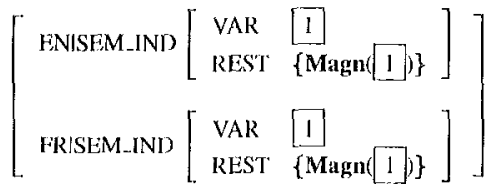

The interlingual status of the lexical function is selfcvident. Any occurrence of Magn will be left intact during transfer and it will be the generation component that ultimately assigns a monolingual lexical entry to the $\mathrm{LH}^{6}$

\subsection{Problems}

I exical Functions abstract away from certain nuances in meaning and from different syntactic realizations. We discuss some of the problems raised by this abstraction in this section.

Overgenerality An important problem stems from the interpretation of LFs implied by their use as an interlingua-- namcly that the meaning of the collocate in some ways reduces to the meaning implied by the lexical function. This interpretation is trouble-free if we assume that LIs always deliver unique values; unfortunately cases to the contrary can be readily observed. An example attested from our corpus was the range of adverbial constructions possible with the verbal head oppose: adamantly, bitterly, consistently, steadfastly, strongly, vehemently, vigorously, deeply, resolutely, etc. The function Magn is an appropriate descriptor in all cases since each adverb functions as a typical intensifier in this context. However each adverb also denotes

\footnotetext{
${ }^{6}$ For more details we refer the reader to (Heylen, 1993). There we also discuss our implenentation in Alep, the C.E.C.'s unification based grammar writing cnvironment.
} 
some other meaning aspect(s). The imprecision of I dis will mean that we have no means of distinguishing between the various intensifiers possible in the context of a given keyword, and hence will not have sufficient information to choose the most appropriate translation where, correspondingly, multiple possibilities exist in the target language. $\Lambda \mathrm{n}$ important question here is how dramatic this loss of translation quality really is.

It is essentially in addressing the issue of overgenerality that Mel'čuk introduces sub- and superscripts to lexical functions, enhancing their precision and making them sensitive to meaning aspects of the lexical items over which they operate. Superscripts are intended to make the meaning of the LF more precise and hence more likely to imply unary mappings between arguments and values, subscripts are used to refcrence a particular semantic component of a keyword. The introduction of such devices into the account of I I is demonstrates both the need for precision and the fact that it does seen necessary to address scmantic aspects of lexemes standing in co-occurrence relations. In fact it has been asserted by some (e.g., (Anick and Pustejovsky, 1990), (IYeid and Ratab, 1989)) that collocational systems ate systematically predictable from the lexical semantics of nouns. In an attempt to explore this notion further, we have investigated the approach to nominal semantics known as Qualia structure (Pustejovsky, 1991) and considered how this may complement the LF notion to improve its descriptive power ${ }^{7}$. Among the promising avenues that oceur to us are, firstly, the postulation of $I I^{i}$ subscripts based on the four Qualia roles (assuming that these are the lexically most relevant aspects of noun semantics) and, secondly, the application of I, 's to semantic (Quatia) structures rather than monolithic lexemes; eg; the L f i lison is used in delivering evaluative qualifiers which are standard expressions of praise or approval. One could imagine application of the function over the Constitutive and $\Lambda$ gentive roles of the nom lecture, to deliver:

$$
\begin{aligned}
& \text { Bon(Const : lecture })=\text { informative } \\
& \text { Bon(Agont: }: \text { lecture })=\text { clear }
\end{aligned}
$$

In both cases the idea is that the precision of the lexical function is essentially enhanced by appealing 10 the semantic facets of its argument.

Syntactic Divergences Another issue that has to be raised concerns the translation of collocations into noncollocational constructions. If we are to maintain a consistent interlingual approach to the translation of these eases, we must extend our Ll-based approach accordingly. We consider one case briefly.

Cross-linguistic analysis reveals many cases where nominal-based collocational constructs are realised as compounds in Germanic languages, c.g., bunch of keys $\Rightarrow$ sleatelbos. A possible account of such phenomena may be developed from the concept of merged I I is (Mel'čuk and $\check{\%}$ olkovsky, 19\%)). Merged LFs are in tended to be used in cases where a value fexeme exists

"lor a comparison between aspects of Oualia structures and lexical functions see (Ileylen, to appeatr). which appears to effectively reduce ("merge") an $\mathrm{LF}$ meaning and its specified argument to a single lexi-. calised form, rather than projecting a syntagmatic unit. We could argue that in cases of compound formation, exactly the same process is to be accounted for, since the compound embodies both the concept mediated by the $\mathrm{LF}^{\mathrm{i}}$ and its argument lexeme. We could therefore allow compounds to be delivered as values of́ nerged I.F's, eg: //Mult(sleutel)= slcutelbos.

T'hese observations are useful in the MT context if we assume that we can elfect a mapping between merged and unmerged I.Fs and therefore capture the correspondence between distinct structural realisations of the same concept. One way to emulate such a mapping might be through the use of Mel'čuk's lexical paraphrasing rales. For instance, one could conceive of a lexical paraphtasing rule as follows $W+\operatorname{Mult}(W) \Leftrightarrow / / \operatorname{Mult}(W)$.

If we assume that in our monolingual Iinglish lexicon, we assign the collocate bunch as the Malt value of keyword key, and that accordingly in the Dutch lexical entry for sleutel we instantiate sleutelbos as the value of the merged I.t: //Mult, then we can use the paraphrasing rule to elfect a mapping between the two LI"s and hence atrive at an interlingual approach to the trans lation of the example, despite structural mismatches, i.e.,

$$
\begin{gathered}
\text { key }+ \text { bunch } \mid \text { Mult }(k e y)] \\
\qquad-\rightarrow \\
\text { sleutelbos[/Mult(sleutel }) \mid
\end{gathered}
$$

lut ther examples exist where productive morphological processes (e.g., affixation") lead to the lexicali. sation in onc language of concepts that exist as syntag. matic constructs in another. Again, we suggest the use of merged I dis and corresponding mappings vial lexical paraphrasing rules as a possible translation strategy in these cases.

\section{Summary and Conclusions}

In this paper we have discussed how the lexicographi cal concept of lexical functions, introduced by Mcl'čuk to describe collocations, can be used as an interlingual device in the machine translation of such structures. We have shown how the essentials of the ICCD analy sis can be embedded in the lexicon and grammar of a unification based theory of language.

Our use of lexical functions as an interlingua assumes that the relevant aspects of the meaning of the collocate are fully captured by the LF. The I, therefore determines the accuracy of translations, which may

\footnotetext{
This is our own in itiative - it seems to he the casc as we examine the literature that neitler L.Fs such as Magn, Bon ete (i.e., those repre senting standard qualificrs/attributes) nor indeed merged 1 t th feature in lexical paraphasing rules. Wo would argue that cross-linguistic analysis suggests that they should enter this domain; compound formation and other types of lexicalisation appear to be regular patterns of translation across many collocational constructs, as we illustrate here.

${ }^{9}$ One could think of an example such as mis-interpret.
} 
be impoverished due to the generalised nature of basic LFs. We have suggested some ways in which LFs can be enriched with lexical semantic information to improve translation quality.

The interlingua level reflects what is semantically common to expressions which form translational equivalents. It abstracts away from specific syntactic realisations. Given that collocations may translate as non-collocations, we also have to provide a way to represent these expressions using lexical functions. We have provided an illustration on how to proceed in one such case.

Acknowledgements We would like to thank the following partners and colleagues: Susan ArmstrongWarwick, Laura Bloksma, Nicoletta Calzolari, R. Lee Humphreys, Simon Murison-Bowie and André Schenk.

\section{References}

[Abeillé and Schabes, 1989] A. Abeillé and Y. Schabes. Parsing idioms in lexicalized tags. In $E A C L / 89$, Manchester, 1989.

[Anick and Pustejovsky, 1990] P. Anick and J. Pustejovsky. An application of lexical semantics to knowledge acquisition from corpora. In Coling/90, Helsinki, 1990.

[Choueka et al., 1983] K. Choueka, S.T. Klein, and E. Neuwitz. Automatic retrieval of frequent idiomatic and collocational expressions in a large corpus. ALLC Journal, pages 34-38, 1983.

[Church and Hanks, 1989] K. W. Church and P. Hanks. Word association norms, mutual information and lexicography. In $A C L / 89$, Vancouver, 1989.

[Heid and Raab, 1989] U. Heid and S. Raab. Collocations in multilingual generation. In EACL/89, pages 130-136, Manchester, 1989

[Heylen, 1993] Dirk Heylen. Collocations and the lexicalisation of semantic operations. Technical report, OTS, 1993.

[Heylen, to appear] D. Heylen. Lexical functions and knowledge representation. In P. Saint-Dizier and E. Viegas, editors, Computational Lexical Semantics. CUP, to appear.

[Krenn and Erbach, to appear] B. Krenn and G. Erbach. Idioms and support verb constructions. In J. Nerbonne, K. Netter, and C. Pollard, editors, German Grammar in HPSG. CSLI Lecture Notes, to appear.

[McRoy, 1992] S. W. McRoy. Using multiple know]edge sources for word sense discrimination. Computational Linguistics, 18(1):1-30, 1992.

[Mel'čuk and Žolkovsky, 1970] I.A. Mel'čuk and A.K. Žolkovsky. Sur la synthèse sémantique. T.A. Informations, 2:1-85, 1970.
[Mel'čuk and Žolkovsky, 1984] I.A. Mel'čuk and A.K. Žolkovsky. Explanatory Combinatorial Dictionary of Modern Russian. Wiener Slawistischer Almanach Sonderband 14, Vienna, 1984.

[Mel'čuk et al., 1984] I. A. Mel'čuk, N. Arbatchewsky-Jumarie, L. Elnitsky, L. Iordanskaja, and A. Lessard. Dictionnaire explicatif et combinatoire du français contemporain. Les Presses de l'Université de Montréal, Montreal, 1984.

[Nirenburg et al., 1988] S. Nirenburg, R. McCardell, E. Nyberg, S. Huffman, E. Kenschaft, and I. Nirenburg. Lexical realization in natural language generation. In Second International Conference on Theoretical and Methodological Issues in Machine Translation of Natural Languages, Pittsburgh, 1988.

[Pollard and Sag, 1987] C. Pollard and I. Sag. Information Based Syntax and Semantics. CSLI, Stanford, 1987.

[Pollard and Sag, to appear] C. Pollard and I. Sag. Head driven phrase structure grammar. to appear.

[Pustejovsky et al., 1992] J. Pustejovsky, S. Bergler, and P. Anick. Lexical semantic techniques for corpus analysis. Computational Linguistics, 19(2):331358, 1992.

[Pustejovsky, 1991] J. Pustejovsky. The generative lexicon. Computational Linguistics, 17(4), 1991.

[Smadja and McKeown, 1990] Frank Smadja and Kathleen R. McKeown. Automatically extracting and representing collocations for language generation. In 28th Annual Meeting of the Association for Computational Linguistics, Pittsburgh, PA, 1990.

[Smadja, 1993] F. Smadja. Retrieving collocations from text: Xtract. Computational Linguistics, 19(1):143-177, 1993. 